\title{
(Non)maximality and Distributivity: A Decision Theory Approach
}

\author{
Sophia A. Malamud \\ University of Pennsylvania
}

\section{Introduction}

\subsection{Vagueness in Questions}

Following Merin's (1999) approach to relevance, van Rooy (1999) uses Decision Theory (DT) to give a formal definition of relevance in its application to questions. In his analysis, questions are inherently underspecified or vague (1): their denotation contains a variable Op (1b) that depends on a decision problem facing the speaker (i.e., the questioner). In the context of the decision problem, vagueness is resolved: a partition is chosen as the true import of the question (1d).

(1) Vagueness in questions: granularity

a. Where do you live?

b. $\{\lambda v[g \in \mathrm{Op}(\mathrm{P})(\mathrm{v})]: \mathrm{w} \in \mathrm{W} \& \mathrm{~g} \in \mathrm{Op}(\mathrm{P})(\mathrm{w})\}, \mathrm{P}=\lambda \mathrm{w} \lambda \mathrm{x}$. You live in $\mathrm{x}$ in $\mathrm{w}$

c. Possible partitions:

i. $\{$ You live in USA, You live in France,,..$\}$

ii. $\left\{\right.$ You live at 1 Sue St. Enid OK, You live at $23^{\text {rd }}$ St. Lodi NJ ... $\}$

d. Goal 1: Questioner interested in background of hearer $\rightarrow$ partition i

Goal 2: Questioner has to mail hearer a package $\quad \rightarrow$ partition ii

The DT approach derives two main types of vagueness: level of granularity as in (1), and degree of exhaustivity (2), where the desired answer could be strongly exhaustive (mention-all) or mention-some.

(2) Where can I buy an Italian newspaper?

\subsection{Vagueness in Plurals}

Striking parallels exist between the types of vagueness inherent in questions and those in definite plurals. First, as repeatedly noted in the literature, definite plurals are vague with respect to the level of granularity (distributivity) (3).

I am grateful to Maribel Romero for providing insight, inspiration, and constant support in discussing this material, to Robin Clark for help with Decision Theory and game-theoretic approaches to language, to Gennaro Chierchia for his incisive criticism making me re-justify and re-examine my framework, and to several anonymous reviewers from various conferences for helpful suggestions on improving the exposition. All faults remain mine. 
(3) a. The boys built a raft.

b. Possible interpretations:

i. Team $1=\{$ Andy,Bill $\}$ built a raft and Team $2=\{$ Chris,Dan $\}$ built a raft ii.Andy built a raft \& Bill built a raft \& Chris built a raft \& Dan...

Second, definites are vague with respect to exhaustivity: (4) is compatible with the maximal (all the windows) or non-maximal construal of the definite, e.g. excepting a few closed windows if the hearer is wondering if his home is stormproof (Krifka 1996).

a. The windows are open.

b. Possible interpretations: i. All of the windows are open

ii. Some of the windows are open

In resolving the vagueness, what factors affect the final interpretation for sentences with definite plurals? First, the nature of the VP plays a role. Many predicates require a certain level of distributivity or collectivity in the subject NP $(5 \mathrm{a}, \mathrm{b})$ (Dowty 1987), while others seem to produce a preference for maximal (5c) or non-maximal (5d) interpretation (Yoon 1996).
a. The boys took a deep breath
b. The boys surrounded the castle
c. The children are healthy
d. The children are sick

While the VP clearly plays a role, it doesn't fully determine the final interpretation - extra-linguistic factors play a role in resolving the vagueness in both distributivity and maximality. Schwarzschild 1991 notes the influence of the extra-linguistic situation on distributivity with (6a, from Schwarzschild 1991); Krifka (1996) shows the effect of context over-riding Yoon's observation on VP influence with (6bc, from Krifka 1996).

(6) a. Situation: Vegetables arrive at a grocery pre-packaged in baskets. The grocery has a big rough scale suitable for small truckloads, and a small scale for weighing only a few veggies or fruits at a time.

Utterance: The vegetables are too light for the big scale and too heavy for the small scale

b. Situation: I am about to travel, and want to ensure my house is safe in my absence.

Utterance: I returned to the house because I thought I had left the windows/doors open

c. Situation: The local bank has a safe that is accessible only through a hallway with three doors, all of which must be open to reach the safe. 
Utterance: I could reach the safe because the doors were open

To refine these observations, note that every scenario in (6) describes, first, an actual salient arrangement of the entities within the NP into 'packages' (veggies pre-packaged in baskets, windows on the outside of the house, doors in a sequence), and also the goals of conversational participants, as spelled out in (7).

a. Utterance: The veggies are too light for the big scale. (excludes collective interpretation)

Packaging: Vegetables arrive at a grocery pre-packaged in baskets.

Goal 1: To double-check the amount delivered $\rightarrow$ Utterance False

Goal 2: To price veggies for sale in baskets $\rightarrow$ Utterance is True

b. Utterance: The windows are open

Packaging: The house has a dozen windows in its outside.

Goal 1: To prepare the house for the arrival of window-frame painters

$\rightarrow$ All windows

Goal 2: To ensure the house is rain-proof before a thunderstorm

$\rightarrow$ Some windows

The empirical data thus indicates the conditions for a theory of interpretation of definite plurals. First, such a theory needs a vague or underspecified semantics that allows for the full range of the collective/distributive and maximal/non-maximal variation. Second, we need a way to encode the effects of the VP, the packaging of entities within the NP denotation, and the speaker/hearer goals in computing the truth-conditions.

The idea that both packaging and speaker/hearer goals play a role in determining interpretation of definite plurals is fairly uncontroversial. Existing accounts, however, have either not spelled out the role of extra-linguistic factors, or focused on the role of packaging.

In the next section, I will sketch three promising approaches to distributivity and maximality in definite plurals, and point to the need for an upgraded account.

\section{Existing Approaches}

\subsection{Landman 1989}

Building on the work of Link (1983), Landman (1989) offers a semantic theory that permits varying levels of distributivity and (non)maximality. He uses the star (*) operator to pluralize VPs, creating distributive interpretations, and permits predicates to distribute over sums of individuals ( $\mathrm{\sqcup} \sqcup \mathrm{b} \sqcup \mathrm{c})$, but not over groups 
$(\uparrow(a \sqcup b \sqcup \mathrm{c})$ or the team of $\mathrm{a}, \mathrm{b}$, and $\mathrm{c})$. All non-maximal interpretations are instances of collective predication (where the plural is interpreted as a group).

Thus, the interpretations for the sentences in $(5 \mathrm{ab}, 6 \mathrm{bc})$ would arise from representations in (8), in a situation where Andy, Bill, Chris, and Dan are the boys, and $\mathrm{d} 1, \ldots \mathrm{d} 5$ are the doors (or windows) (following the arrow I include a simplified formula that indicates more precisely a situation in which the interpretation is true). Note that the only way to require a fully maximal interpretation is to make it fully distributive (8d).

a. $\llbracket 5 \mathrm{a} \rrbracket=\llbracket$ The boys took a deep breath $\rrbracket=$ $=\lambda \mathrm{w} \cdot \exists \mathrm{e}^{*}$ TOOK.A.DEEP.BREATH $(\mathrm{e}, \mathrm{w}) \& * \operatorname{Ag}(\mathrm{e})=\sigma \mathrm{x} * \operatorname{boy}(\mathrm{x}, \mathrm{w}) \rightarrow$ $\rightarrow(\mathrm{a} \sqcup \mathrm{b} \sqcup \mathrm{c} \sqcup \mathrm{d}) \in{ }^{*}$ TOOK.A.DEEP.BREATH(w)

b. $\llbracket 5 \mathrm{~b} \rrbracket=\llbracket$ The boys surrounded the castle $\rrbracket=$ $=\lambda \mathrm{w} \cdot \exists \mathrm{e}^{*} \operatorname{SurRoundED} \cdot \operatorname{THE} \cdot \operatorname{CASTLE}(\mathrm{e}, \mathrm{w}) \& * \operatorname{Ag}(\mathrm{e})=\uparrow \sigma \mathrm{x} * \mathrm{boy}(\mathrm{x}, \mathrm{w}) \rightarrow$ $\rightarrow \uparrow(\mathrm{a} \sqcup \mathrm{b} \sqcup \mathrm{c} \sqcup \mathrm{d}) \in \operatorname{SURROUNDED}$.THE.CASTLE$(\mathrm{w}) \quad$ collective

c. $\llbracket 6 \mathrm{~b} \rrbracket=\llbracket$ The doors are open $\rrbracket=$ $=\lambda \mathrm{w} \cdot \exists \mathrm{e}^{*} \operatorname{OPEN}(\mathrm{e}, \mathrm{w}) \& * \operatorname{Ag}(\mathrm{e})=\uparrow \sigma \mathrm{x} * \operatorname{door}(\mathrm{x}, \mathrm{w}) \rightarrow$ $\rightarrow \uparrow(\mathrm{d} 1 \sqcup \mathrm{d} 2 \sqcup \mathrm{d} 3 \sqcup \mathrm{d} 4 \sqcup \mathrm{d} 5) \in \mathrm{OPEN}(\mathrm{w})^{1}$ collective

d. $\llbracket 6 \mathrm{c} \rrbracket=\llbracket$ The doors are open $\rrbracket=$ $=\lambda \mathrm{w} \cdot \exists \mathrm{e} * \operatorname{OPEN}(\mathrm{e}, \mathrm{w}) \& * \operatorname{Ag}(\mathrm{e})=\sigma \mathrm{x}$ door $*(\mathrm{x}, \mathrm{w}) \rightarrow$ $\rightarrow(\mathrm{d} 1 \sqcup \mathrm{d} 2 \sqcup \mathrm{d} 3 \sqcup \mathrm{d} 4 \sqcup \mathrm{d} 5) \in *^{*} \mathrm{OPEN}(\mathrm{w})$

\section{distributive}

It is not clear whether Landman's original 1989 theory allows for the intermediate-distributive interpretations like (6a), repeated below.

However, his 1996 paper explicitly derives them via adaptation of Schwarzschild's 1991 proposal described below in section 2.3 and exemplified in (8e), where artichoke, Brussels sprout, cauliflower, daikon, and endive are the vegetables. The definition of 'cover-agent' ${ }^{\mathrm{c}} \mathrm{Ag}(\mathrm{e})$ is given in section 2.3.

e. $\llbracket 6 \mathrm{a} \rrbracket=\llbracket$ The vegetables are too light for the big scale $\rrbracket=$ $=\lambda \mathrm{w} \cdot \exists \mathrm{e} *$ TOO-Light $(\mathrm{e}, \mathrm{w}) \&{ }^{\mathrm{c}} \operatorname{Ag}(\mathrm{e})=\uparrow \sigma \mathrm{x} \operatorname{veg}^{*}(\mathrm{x}, \mathrm{w}) \rightarrow$ $\rightarrow(\uparrow(\mathrm{a} \sqcup \mathrm{b}) \sqcup \uparrow(\mathrm{c} \sqcup \mathrm{d} \sqcup \mathrm{e})) \in^{*} \mathrm{TOO}-\mathrm{LIGHT}(\mathrm{w})$ intermediate-distributive

\footnotetext{
${ }^{1}$ I am allowing here, counter-intuitively, that predicates like 'open' or 'take a breath,' do not have a built-in lexical requirement that they must distribute fully down to individuals. Then, Landman 1989 and 1996 can derive non-maximal interpretations for 'open' (or 'take a breath') by giving the windows or doors a collective responsibility for the state of being open. If instead we say that selectional requirements of 'open' and 'take a breath' demand full distributivity, then neither Landman 1989 nor the theory described in section 2.3 below can derive non-maximal readings, and a pragmatic mechanism is necessary, like the one in Brisson 1998 or the one I propose below.
} 
Landman's theory posits a pervasive ambiguity between singular and plural VPs, and does not distinguish between the maximal and non-maximal interpretations in the representation (cf. $8 \mathrm{~b}$ and $8 \mathrm{c}$ ). ${ }^{2}$

Even more importantly for us, as can be seen from (8), the theory does not provide an explanation of how and when the various interpretations arise, since it does not include an account of pragmatic factors (whether the structure of a situation or the intent of a conversational agent).

\subsection{Schwarzschild (1991) and Brisson (1998)}

Schwarzschild (1991) proposes an account of distributivity, focusing on spelling out the role of packaging by building a free variable over covers $\left(\mathrm{Cov}_{\mathrm{i}}\right)$ into the denotation of VPs, which allows them to distribute up to sub-pluralities of the definite plural given by the cover. A cover is defined in (9a); the specific cover in (9b) derives the intermediate-distributive interpretations (6c, 9c) from the representation in $(9 d)$.

(9) a. Cover: a set of sets of entities, such that the union of the sets in the cover is the universe of discourse.

b. $\{\{$ arti1,arti2,Br.sprout1,Br.sprout 2$\},\{$ caulif,daikon,endive $\},\{$ John... $\}\}$

c. The vegetables are too heavy for the big scale and too light for the small scale

d. $\forall \mathrm{x}\left[\mathrm{x} \in \llbracket \operatorname{Cov}_{\mathrm{i}} \rrbracket \& \mathrm{x} \subseteq \llbracket\right.$ the.veg' $\rrbracket \mapsto \mathrm{x} \subseteq \llbracket$ too.heavy.for.sc1\&too.light.for.sc2' $\left.\rrbracket\right]$

Brisson (1998) builds on Schwarzschild (1991) to permit exceptions by allowing mismatch between the distribution of individuals into the cover-cells and the NP denotations (ill-fitting covers (defined in 10a)).

Unlike Landman (1989), she only derives cases of a few salient exceptional items (10c, from representation in 10d), rather than existential interpretations like $(6 b, 7 b-2)$.

(10) a. A cover is ill-fitting with respect to an NP denotation if some members of NP denotation are in the same cover-cell as non-members, so no union of cells in the cover equals the NP denotation.

b. $\{\{$ windw 1$\},\{$ windw 2$\},\{$ windw 3$\},\{$ windw 4$\},\{$ windw5,Mary, door $\}\}$

c. The windows are open (but we didn't get to the bathroom window yet)

d. $\forall \mathrm{x}\left[\mathrm{x} \in \llbracket \operatorname{Cov}_{\mathrm{i}} \rrbracket \& \mathrm{x} \subseteq \llbracket\right.$ the.windows' $\rrbracket \rightarrow \mathrm{x} \subseteq \llbracket$ open' $\left.\rrbracket\right]$

There are serious drawbacks in the cover-based account.

\footnotetext{
${ }^{2}$ This inability to distinguish between maximal and non-maximal interpretations in theory matches empirical data for collective predicates: since the plural agent bears collective responsibility for the action, we cannot distinguish whether all or some of the agent's subparts actually participated.
} 
First, the final interpretation depends on the distribution of individuals into cover-cells, making non-maximality essentially a matter of narrowing the domain to a relevant set. In this, the cover-based account of Brisson 1998 inherits the problem of any theory that derives non-maximality through domain narrowing.

In fact, as examples $(6 \mathrm{~b}, 7 \mathrm{~b})$ repeated below (11) demonstrate, nonmaximality is not a matter of domain selection of any kind: there is no "relevant set" of open windows, and no window is more relevant than any other - it is simply irrelevant which or how many windows are open, as long as some of them are. This problem is intrinsic in the cover-based approach, and derives incorrect interpretations for many non-maximal uses of definite plurals.

(11) Scenario: The house has a dozen windows on the outside. The hearer and speaker are going on a trip and want to make sure the house is safe.

Utterance: The windows are open

Cover-derived interpretation: The relevant/salient windows are open

Actually: Some of the windows are open (it doesn't matter which ones, and the speaker may not know which ones)

Second, the distribution of individuals into cover-cells is denoted by a free, deictic variable, whose denotation is fixed when a particular cover is made salient in preceding discourse or extra-linguistic context. This deictic nature of $\mathrm{Cov}_{\mathrm{i}}$ requires at least the speaker to know the exact composition of cover-cells, including the special cell containing exceptions, in the same way this is required for a pronoun.

This is strikingly contrary to fact: the speaker doesn't need to know the identity of the vegetables for the intermediate distributivity interpretation to arise in $(6 \mathrm{c}, 9 \mathrm{c})$, nor does he need to know the identity of the exceptional windows for the non-maximal interpretation to obtain in $(4,6 \mathrm{~b}, 7 \mathrm{~b})$.

One way to avoid making demands on speaker knowledge is to intensionalize the $\operatorname{Cov}_{\mathrm{i}}$ variable, varying the distribution of individuals into cells with each possible world compatible with what the speaker knows. As Kratzer (2003) notes, "amending Schwarzschild's account of plural predication, we would want to say that plural predication depends on contextually provided cover functions, not just on contextually provided covers."

In terms of the needs outlined in the previous section, the cover account derives (at least some of) the influence of the VP (since the $\operatorname{Cov}_{i}$ variable is part of the VP) and of the "packaging" aspect of scenarios (whenever context provides a salient cover that yields correct interpretation), but does not have a mechanism for integrating the influence of speaker/hearer goals into the analysis. 


\subsection{Landman (1996) and His Recasting of Schwarzschild (1991)}

In his wide-reaching article "Plurality", Landman (1996) provides a recasting of Schwarzschild's 1991 proposal in his own framework (what he dubs 'Theory IV' of plurality). This is done by allowing cover roles, defined as in (12a), and illustrated in the proof (12d) for the denotation (12c) for the sentence (12b), in a situation where Andy, Bill, Chris, and Dan are the boys (compare with 6c, 9c).

a. Definition: Let $\mathrm{R}$ be a thematic role. Then ${ }^{\mathrm{c}} \mathrm{R}$, the cover role based on $R$, is the partial function from $D_{e}$ (domain of events) into $D_{d}$ (domain of individuals) defined by

${ }^{\mathrm{c}} \mathrm{R}(\mathrm{e})=\mathbf{a}$ iff $\mathbf{a} \in \mathrm{ATOM} \& \sqcup(\{\downarrow(\mathrm{d}) \in \mathrm{SUM}: \mathrm{d} \in \mathrm{AT}(* \mathrm{R}(\mathrm{e}))\})=\downarrow(\mathbf{a})$

Paraphrase: $\mathrm{a}$ is either a singular atom or a group, and the set underlying $\mathbf{a}$ is the union of the elements underlying atomic subparts of the plural role based on $\mathrm{R}$.

b. The boys built a raft (in a situation where there are two teams of boys)

c. $\lambda w . \exists e{ }^{*}$ Built.A.RAFT $(\mathrm{e}, \mathrm{w}) \&{ }^{\mathrm{c}} \mathrm{Ag}(\mathrm{e})=\uparrow \sigma \mathrm{x} * \operatorname{boy}(\mathrm{x}, \mathrm{w}) \rightarrow$

$\rightarrow \lambda \mathrm{w} . \uparrow(\mathrm{a} \sqcup \mathrm{b}) \sqcup \uparrow(\mathrm{c} \sqcup \mathrm{d}) \in{ }^{*} \mathrm{BUILT}-\mathrm{A}-\mathrm{RAFT}(\mathrm{w})$

d. $\quad \mathrm{e}=\mathrm{f} \sqcup \mathrm{g}, \operatorname{Ag}(\mathrm{f})=\uparrow(\mathrm{a} \sqcup \mathrm{b}), \operatorname{Ag}(\mathrm{g})=\uparrow(\mathrm{c} \sqcup \mathrm{d})$; then $* \operatorname{Ag}(\mathrm{e})=\uparrow(\mathrm{a} \sqcup \mathrm{b}) \sqcup \uparrow(\mathrm{c} \sqcup \mathrm{d})$

$\{\downarrow(\mathrm{d}): \mathrm{d} \in \mathrm{AT}(* \mathrm{R}(\mathrm{e}))\}=\{\mathrm{a} \sqcup \mathrm{b}, \mathrm{c} \sqcup \mathrm{d}\}$, and $\sqcup(\{\downarrow(\mathrm{d}): \mathrm{d} \in \mathrm{AT}(* \mathrm{R}(\mathrm{e}))\})=$

$=\sqcup(\{\mathrm{a} \sqcup \mathrm{b}, \mathrm{c} \sqcup \mathrm{d}\})=\mathrm{a} \sqcup \mathrm{b} \sqcup \mathrm{c} \sqcup \mathrm{d}=\downarrow(\uparrow \sigma \mathrm{x} * \operatorname{boy}(\mathrm{x}, \mathrm{w})) \quad$ q.e.d.

The cover roles are a very powerful mechanism: using them, we can derive the interpretations in (5ab, 6abc) in a similar way (13).

a. $\llbracket 5 \mathrm{a} \rrbracket=\llbracket$ The boys took a deep breath $\rrbracket=$

$=\lambda \mathrm{w} \cdot \exists \mathrm{e}^{*}$ TOOK.A.DEEP.BREATH $(\mathrm{e}, \mathrm{w}) \&{ }^{\mathrm{c}} \operatorname{Ag}(\mathrm{e})=\sigma \mathrm{x} * \operatorname{boy}(\mathrm{x}, \mathrm{w})$

$\mathrm{e}=\mathrm{f} \sqcup \mathrm{g} \sqcup \mathrm{h} \sqcup \mathrm{i}, \operatorname{Ag}(\mathrm{f})=\mathrm{a}, \operatorname{Ag}(\mathrm{g})=\mathrm{b}, \operatorname{Ag}(\mathrm{h})=\mathrm{c}, \operatorname{Ag}(\mathrm{i})=\mathrm{d}$,

then *Ag(e)=a $\sqcup \mathrm{b} \sqcup \mathrm{c} \sqcup \mathrm{d}$, so $\sqcup(\{\downarrow(\mathrm{d}): \mathrm{d} \in \mathrm{AT}(* \mathrm{R}(\mathrm{e}))\})=$

$=\sqcup(\{\mathrm{a}, \mathrm{b}, \mathrm{c}, \mathrm{d}\})=\mathrm{a} \sqcup \mathrm{b} \sqcup \mathrm{c} \sqcup \mathrm{d}=\downarrow(\uparrow \sigma \mathrm{x} * \operatorname{boy}(\mathrm{x}, \mathrm{w}))$

q.e.d.

b. $\llbracket 5 \mathrm{~b} \rrbracket=\llbracket$ The boys surrounded the castle $\rrbracket=$

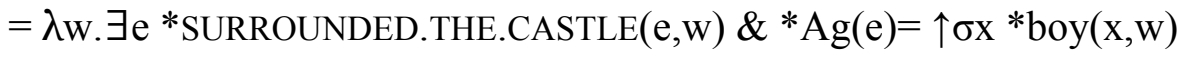

e is atomic, $* \operatorname{Ag}(\mathrm{e})=\uparrow(\mathrm{a} \sqcup \mathrm{b} \sqcup \mathrm{c} \sqcup \mathrm{d})$, so $\sqcup(\{\downarrow(\mathrm{d}): \mathrm{d} \in \mathrm{AT}(* \mathrm{R}(\mathrm{e}))\})=$

$=\sqcup(\{\mathrm{a} \sqcup \mathrm{b} \sqcup \mathrm{c} \sqcup \mathrm{d}\})=\mathrm{a} \sqcup \mathrm{b} \sqcup \mathrm{c} \sqcup \mathrm{d}=\downarrow(\uparrow \sigma \mathrm{x} * \operatorname{boy}(\mathrm{x}, \mathrm{w})) \quad$ q.e.d.

c. $\llbracket 6 \mathrm{~b} \rrbracket=\llbracket$ The doors/windows are open $\rrbracket=$

$=\lambda \mathrm{w} . \exists \mathrm{e} * \operatorname{OPEN}(\mathrm{e}, \mathrm{w}) \& * \operatorname{Ag}(\mathrm{e})=\uparrow \sigma \mathrm{x} * \operatorname{door}(\mathrm{x}, \mathrm{w})$ (see footnote 1 )

e is atomic, ${ }^{*} \operatorname{Ag}(\mathrm{e})=\uparrow(\mathrm{d} 1 \sqcup \mathrm{d} 2 \sqcup \mathrm{d} 3 \sqcup \mathrm{d} 4 \sqcup \mathrm{d} 5)$, so $\sqcup(\{\downarrow(\mathrm{d})\})=$

$=\sqcup(\{\mathrm{d} 1 \sqcup \ldots \sqcup \mathrm{d} 5\})=\mathrm{d} 1 \sqcup \mathrm{d} 2 \sqcup \mathrm{d} 3 \sqcup \mathrm{d} 4 \sqcup \mathrm{d} 5=\downarrow(\uparrow \sigma \mathrm{x} *$ door $(\mathrm{x}, \mathrm{w}))$ q.e.d.

d. $\llbracket 6 \mathrm{c} \rrbracket=\llbracket$ The doors are open $\rrbracket=$

$=\lambda \mathrm{w} \cdot \exists \mathrm{e} * \operatorname{OPEN}(\mathrm{e}, \mathrm{w}) \& * \operatorname{Ag}(\mathrm{e})=\sigma \mathrm{x}$ door $*(\mathrm{x}, \mathrm{w})$

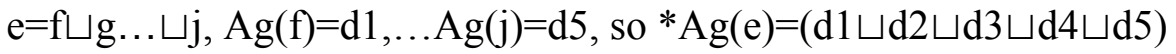

so $\sqcup(\{\downarrow(\mathrm{d})\})=\sqcup(\{(\mathrm{d} 1, \ldots, \mathrm{d} 5)\})=\mathrm{d} 1 \sqcup \ldots \sqcup \mathrm{d} 5=\downarrow(\uparrow \sigma \mathrm{x} * \operatorname{door}(\mathrm{x}, \mathrm{w}))$ q.e.d. 
Landman's reinterpretation preserves the flexibility of Schwarzschild's theory, allowing for the intermediate-distributive interpretations like (6c, 9c, 12d). Representation no longer contains the free, deictic variable $\mathrm{Cov}_{\mathrm{i}}$.

On this approach, the speaker only has to know that there is a cover making the statement true (i.e., the speaker must only be sure of the existence of a proof like the one in (12b), where knowledge that the teams are boy-made will point to the existence of such a proof).

The resulting analysis allows the full range of empirically attested interpretations, and says nothing about the way these various interpretations are derived and the factors that influence the derivation.

Sentences like (14) just mean that subparts of the given group of windows/boys, in some packaging, are agents for (potentially plural) states of being open / events of raft-building.
a. The windows are open
b. The boys built a raft

This semantics is very weak. The only information transmitted with the use of a cover-role is that there exists some proof like the ones in $(12,13)$ - so, this incorrectly predicts that in a situation (6c), repeated below (15), the speaker can say The doors are open on the collective interpretation (13c), allowing some doors to be actually closed, since a proof exists as long as some doors are open.

(15) Scenario: The hearer works in a bank and must get to a safe that can only be reached via three consecutive doors.

Utterance: The doors are open

Interpretation allowed in Theory IV: Some/All of the doors are open Actual interpretation: All of the doors are open

This is much less information than would actually be transmitted by such an utterance in the situation (15).

Similarly, this account incorrectly predicts that in a situation from (6a), repeated below in (16), the speaker can truthfully utter 'The vegetables are not too light for the big scale' on the collective interpretation, even when the baskets are actually too light for the scale. After all, the possibility of putting all the baskets on the scale at once points to the existence of some way to make the sentence true.

(16) Scenario: The vegetables arrive to the grocery store pre-packaged in baskets, and need to be priced by weight. The store has a scale suitable for only for truckloads of stuff.

Utterance: The vegetables are not too light for the big scale Interpretation allowed in Theory IV: The veggies, possibly weighed all 
at once, are not too light for the big scale

Actual interpretation: The veggies, weighed by the basket, are not too light for the big scale.

Thus, by getting rid of the deictic cover variable, we have lost crucial information in this case.

While clearly too weak to stand on its own, this semantics is the perfect starting point for an account drawing on pragmatic factors to derive a stronger interpretation - after all, in a particular scenario $(6,7)$, hearers are perfectly clear on whether all or some windows/doors are required to be open, and on which packaging is meant when baskets of vegetables need to be weighed.

\subsection{Interim Conclusions}

In a theory of definite plurals, we must take into account that in accounting for the influence of packaging on interpretation, deixis to packaging is necessary in some cases (e.g., 6a,16) - without it, the semantics is too weak $(15,16)$. At the same time, deixis to packaging is wrong in some cases $(6 \mathrm{~b}, 11)$ - because it makes non-maximality a matter of domain selection, and so the interpretations derived using it are contrary to fact.

We still need to incorporate speaker/hearer goals into the analysis.

In the proposal I lay out in the next section, I will introduce a deictic variable that will always include reference to hearer's goals, and will encode information about packaging only when such information is relevant to hearer's goals.

\section{Decision Theory Approach}

\subsection{The Proposal}

I apply Merin's (1999) and van Rooy's (1999) Decision Theory (DT) based definition of relevance to definite plurals, replacing the cover analysis, integrating Landman's (1996) recasting of Schwarzschild's proposal with a principled account of how and when the various interpretations arise.

The chief innovation is thus the unification of the vague/flexible semantics with a formal analysis of pragmatic factors influencing the truth-conditions of sentences with definite plurals.

Agents in conversation are constantly modeling each other's goals. As a part of cooperative communication, each speaker aims to change the hearers' states of knowledge so as to help them progress towards their goals. This is the heart of the Cooperative Principle (Grice 1975), and in particular, of the Relation (Relevance) maxim. 
When interpreting a vague utterance (in this case, one containing a definite plural), hearers select propositions which can influence their actions in achieving the goal. Agents' goals (and more) can be represented as decision problems (DeP) they are solving. A DeP is a triple $<\mathrm{P}, \mathrm{U}, \mathrm{A}>$, where the probability function $P$ represents agent's beliefs, utility function $U$ reflects the agent's preferences, and a set of (mutually exclusive) actions A the agent chooses from.

A proposition $q$ changes agent's beliefs $(\mathrm{P})$, resolving the DeP if, after $q$ is learned, a single action has, in each resulting world, the highest utility. In making an utterance, the speaker aims to resolve hearers' $\mathrm{DeP}^{3}$.

A relevance ordering between propositions (17) yields the contextual criterion for licensing and choosing an interpretation for plural definites, just as it does for questions, with relevance defined as helpfulness in resolving the DeP.

\section{(17) Proposition $p$ is more relevant (better to learn) for resolving DeP than $q$ $\left(p>_{D e P} q\right)$ iff \\ i. $p$ eliminates more actions as non-optimal than $q$ does or \\ ii. $p$ eliminates the same number of actions as $q$ does, and $q$ entails $p$ \\ (i.e. $q$ is over-informative)}

This relevance ordering, built into the definition of the variable REL (18) ${ }^{4}$, allows the hearer to choose an appropriate interpretation for the vague definite. ${ }^{5}$

(18) The relevance operator:

a. Definition: $\operatorname{REL}(\mathrm{DeP})(\mathrm{VP})(\mathrm{NP})(\mathrm{w})=$

$$
\begin{aligned}
& \{\mathrm{g}: \sqcup\{\downarrow \mathrm{d}: \mathrm{d} \in \mathrm{AT}(\downarrow \mathrm{g})\} \subseteq \downarrow \mathrm{NP}(\mathrm{w}) \& \neg \exists \mathrm{h}[\sqcup\{\downarrow \mathrm{d}: \mathrm{d} \in \mathrm{AT}(\downarrow \mathrm{h})\} \subseteq \downarrow \mathrm{NP}(\mathrm{w}) \\
& \left.\left.\&\left[\lambda \mathrm{w} \cdot \operatorname{VP}(\mathrm{e}, \mathrm{w}) \&{ }^{*} \operatorname{Ag}(\mathrm{e})=\mathrm{h}>_{\operatorname{DeP}} \lambda \mathrm{w} \cdot \operatorname{VP}(\mathrm{e}, \mathrm{w}) \&^{\mathrm{c}} \operatorname{Ag}(\mathrm{e})=\mathrm{g}\right]\right]\right\}
\end{aligned}
$$

Paraphrase: REL is a function that takes the decision problem, VP and NP denotations, and a world, and outputs a set of individuals $\mathbf{g}$, which could be groups or sums.

The framed conjunct assures that when you boil g down to singularities, all of those singular individuals are atoms in the NP denotation (e.g., 'the boys').

The second conjunct assures that no other such NP-made (e.g., boymade) thing $\mathbf{h}$ is more relevant than $\mathbf{g}-$ i.e., there is no $\mathbf{h}$ such that, as the underlined portion states, it's better to learn that " $h$ VPs" than that "g VPs."

\footnotetext{
${ }^{3} \mathrm{Or}$, rather, the speaker aims to resolve his/her estimate or hearer's DeP. While practically all linguists agree that conversational participants represent each other's goals and knowledge during a conversation, the processes and representations utilized during such modeling are subject of much ongoing research. For the purposes of this paper and of (18), it is enough if speakers have an idea about the actions their hearers are choosing from, and can figure out the effect of learning various propositions on that action set.

${ }^{4} \mathrm{REL}$ for 'relevant,' instead of a similar variable Op proposed by van Rooy.

${ }^{5}$ If $\mathrm{x}$ is an atom, $\downarrow \mathrm{x}=\mathrm{x}$ (Landman 1989). If $\mathrm{x}$ a sum, rather than an atom, I assume $\downarrow \mathrm{x}=\mathrm{x}$.
} 
REL replaces Schwarzschild's (1991) $\operatorname{Cov}_{\mathrm{i}}$ in encoding the vagueness and context-dependence. REL operator comes as part of the VP, following syntactic assumptions in Schwarzschild (1991) and preceding literature on distributivity (196c). The operator containing REL introduces a set of alternatives $-\mathrm{O}_{\mathrm{Rel}}$ is a function from VP and NP denotations to the set of optimally relevant propositions (196a). The sentential existential quantifier converts the set into a single proposition at the top. This last operation is the one introduced for the Hamblin semantics in Kratzer and Shimoyama (2002) (see also Keshet (this volume)). The schema for the entire sentence is given in (19).

(19) The relevance operator (continued):

a. $\mathrm{O}_{\mathrm{Rel}}=\lambda \mathrm{w} . \lambda \mathrm{P} . \lambda \mathrm{q} . \lambda \mathrm{x} \lambda \mathrm{e} . \mathrm{x} \in \mathrm{REL}(\mathrm{DeP})(\mathrm{P})(\mathrm{q})(\mathrm{w}) \& \mathrm{P}(\mathrm{e}, \mathrm{w}) \& * \operatorname{Ag}(\mathrm{e}, \mathrm{x})$

b. $\lambda \mathrm{w} . \exists \mathrm{p}\left[\mathrm{p} \in\left\{\lambda \mathrm{v} \cdot\left[\mathrm{g} \in \mathrm{REL}(\mathrm{DeP})(\mathrm{VP})(\mathrm{NP})(\mathrm{v}) \& \mathrm{VP}(\mathrm{e}, \mathrm{v}) \&^{*} \operatorname{Ag}(\mathrm{e})=\mathrm{g}\right]\right.\right.$ :

$: \mathrm{g} \in \mathrm{REL}(\mathrm{DeP})(\mathrm{VP})(\mathrm{NP})(\mathrm{w})\}$ \&

$\mathrm{p}(\mathrm{w})=1]$

'There is a true proposition, taken from the set of optimally-relevant propositions saying that some subpart(s) of the NP do the VPing.'

c. $\lambda \mathrm{w} . \exists \mathrm{p}\left[\mathrm{p} \in\left\{\lambda \mathrm{v} \cdot\left[\mathrm{g} \in \mathrm{REL}(\mathrm{DeP})(\mathrm{VP})(\mathrm{NP})(\mathrm{v}) \& \mathrm{VP}(\mathrm{e}, \mathrm{v}) \&^{*} \mathrm{Ag}(\mathrm{e})=\mathrm{g}\right]\right.\right.$ :

$\mathrm{p}(\mathrm{w})=1]$

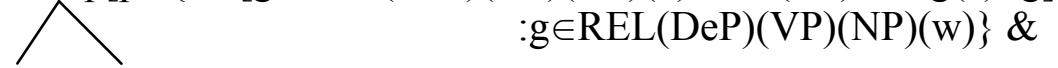

$\exists \quad \mathrm{IP}=\lambda \mathrm{w} \cdot \lambda \mathrm{x} . \lambda \mathrm{e} . \mathrm{x} \in \mathrm{REL}(\mathrm{DeP})(\mathrm{VP})(\mathrm{NP})(\mathrm{w}) \& \mathrm{VP}(\mathrm{e}, \mathrm{w}) \& * \operatorname{Ag}(\mathrm{e}, \mathrm{x})$

$\mathrm{VP}=\lambda \mathrm{w} \cdot \lambda \mathrm{q} \cdot \lambda \mathrm{x} \cdot \lambda \mathrm{e} \cdot \mathrm{x} \in \mathrm{RED}(\operatorname{DeP})(\mathrm{VP})(\mathrm{q})(\mathrm{w}) \& \mathrm{VP}(\mathrm{e}, \mathrm{w}) \& * \operatorname{Ag}(\mathrm{e}, \mathrm{x})$

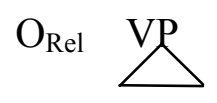

\subsection{Deriving (Non)Maximality}

I provide several worked-out examples to illustrate the schema in (19) in deriving maximal and non-maximal interpretations for definite plurals. ${ }^{6}$

(20) a. Decision Problem: Before a thunderstorm, Hearer has to decide whether to go on with daily business (Action1) if all windows are closed or return home (Action2) if some windows are open.

b. Utterance: The windows are closed $=$ $=\lambda \mathrm{w} . \exists \mathrm{p}[\mathrm{p} \in\{\mathrm{g}(\mathrm{w}) \in \mathrm{REL}(\operatorname{DeP})(\mathrm{win})(\mathrm{clo})(\mathrm{w}) \& \mathrm{~g}(\mathrm{w})$ is closed $\} \& \mathrm{p}=1]$

c. Pool of propositions REL chooses from:

$\mathrm{w} 1 \sqcup \mathrm{w} 2 \sqcup \mathrm{w} 3$ is closed $\hat{\uparrow}(\mathrm{w} 1 \sqcup w 2 \sqcup w 3)$ is closed $\hat{f}(w 1 \sqcup w 2)$ closed $\hat{\uparrow}(w 2 \sqcup w 3)$ closed $\hat{\uparrow}(w 1 \sqcup w 3)$ closed $\hat{\uparrow}(w 1 \sqcup w 2) \sqcup w 3$ is closed ...

\footnotetext{
${ }^{6}$ In (20), the propositions with a single strike-through don't resolve the DeP, and are eliminated by REL.
} 
$\uparrow(w 1 \sqcup w 2) \sqcup \uparrow(w 1 \sqcup w 3)$ is closed...

$w 1 \sqcup w 2$ closed $w 2 \sqcup w 3$ closed $\quad w 1 \sqcup w 3$ is closed

$w 1$ is closed $w 2$ is closed w3 is closed

\section{d. Some possible interpretations:}

(:) i. $\exists \mathrm{p}\left[\mathrm{p} \in\left\{\exists \mathrm{e} \cdot{ }^{*} \mathrm{clo}(\mathrm{e}) \& \mathrm{w} 1+\mathrm{w} 2+\mathrm{w} 3+\mathrm{w} 4+\mathrm{w} 5=* \operatorname{Ag}(\mathrm{e})\right\} \& \mathrm{p}=1\right]$

(all of the windows are closed)

ii.

$\exists \mathrm{p}\left[\mathrm{p} \in\left\{\exists \mathrm{e}^{*} \mathrm{clo}(\mathrm{e}) \& \mathrm{w} 1=* \operatorname{Ag}(\mathrm{e}), \exists \mathrm{e} * \operatorname{clo}(\mathrm{e}) \& \mathrm{w} 2=* \operatorname{Ag}(\mathrm{e}), \ldots\right\} \& \mathrm{p}=1\right]$

(some of the windows are closed)

Consider an agent facing the problem in (20a). If he hears (20b), he has to select one of the interpretations [i] or [ii] as the true import of the vague literal statement. While [i] resolves the decision problem (by pointing to Action1), [ii] fails to do so, since it is compatible both with a scenario where all windows are closed, and one in which some are open and some closed. So, [i] is the only relevant interpretation.

A built-in consequence of the framework is this: the only way to force a maximal interpretation is for REL to produce a single most-relevant proposition, so that the existential quantification over propositions is over a singleton set. Further, we inherit from Landman the fact that maximal interpretations are simply fully distributive ones, where a unique optimal proposition involves distributive predication over a sum.

To illustrate what happens when these conditions fail to hold, consider the example in (21) below. ${ }^{7}$

(21) a. Decision Problem: Hearer is preparing his house for arrival of painters,

who will paint all the window-frames. He has to decide if he can relax till they arrive (Action 1), or if he still needs to do something to prepare for the painters (Action 2).

b. Utterance - same as in (20b): The windows are closed $=$ $=\lambda \mathrm{w} . \exists \mathrm{p}[\mathrm{p} \in\{\mathrm{g}(\mathrm{w}) \in \mathrm{REL}(\operatorname{DeP})(\mathrm{win})(\mathrm{clo})(\mathrm{w}) \& \mathrm{~g}(\mathrm{w})$ is closed $\} \& \mathrm{p}=1]$

c. Pool of propositions REL chooses from (see footnotes $1,5,6,7$ )

$\uparrow(\mathrm{w} 1 \sqcup \mathrm{w} 2 \sqcup \mathrm{w} 3)$ is closed

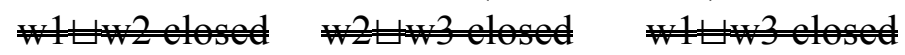

$\uparrow(\mathrm{w} 1 \sqcup \mathrm{w} 2)$ closed $\uparrow(\mathrm{w} 2 \sqcup \mathrm{w} 3)$ closed $\uparrow(\mathrm{w} 1 \sqcup \mathrm{w} 3)$ closed

$\mathrm{W} 1$ is closed $\quad \mathrm{W} 2$ is closed $\mathrm{W} 3$ is closed

$\hat{f}(1 \pm 2) \sqcup 3$ i

d. Some possible interpretations:

i. $\exists \mathrm{p}\left[\mathrm{p} \in\left\{\exists \mathrm{e}{ }^{*} \operatorname{clo}(\mathrm{e}) \& \mathrm{w} 1+\mathrm{w} 2+\mathrm{w} 3+\mathrm{w} 4+\mathrm{w} 5=* \operatorname{Ag}(\mathrm{e})\right\} \& \mathrm{p}=1\right]$

\footnotetext{
${ }^{7}$ Propositions with a double strike-through are over-informative and so are eliminated by REL.
} 
(all of the windows are closed)

(:) ii. $\exists \mathrm{p}\left[\mathrm{p} \in\left\{\exists \mathrm{e}^{*} \operatorname{clo}(\mathrm{e}) \& \mathrm{w} 1=* \operatorname{Ag}(\mathrm{e}), \exists \mathrm{e}^{*} \operatorname{clo}(\mathrm{e}) \& \mathrm{w} 2=* \operatorname{Ag}(\mathrm{e}), \ldots\right\} \& \mathrm{p}=1\right]$ (some of the windows are closed)

Consider an agent facing a different problem (21a). If he hears the same utterance as before $(20 \mathrm{~b}, 21 \mathrm{~b})$, then both [i] and [ii] resolve the DeP (by pointing to Action2) and thus are both relevant. But [i] entails [ii] and thus is overinformative (hence less relevant according to (17)). This points to [ii] as the intended message.

Thus, whenever REL fails to winnow the set down to a single fullydistributive proposition, the existential quantification over propositions will result in a weaker meaning, stating that one of the most-helpful propositions is true. In the case when the hearer doesn't care which windows are closed, all propositions stating that one of the windows is closed are equally most-helpful. Thus, the existential quantification over propositions creates the effect of existential quantification over the windows, i.e., the non-maximal reading.

\subsection{Deriving Distributivity}

The worked example below illustrates that the hearer chooses the level of distributivity based on (17) in a (by now) familiar fashion.

(22) a. Decision Problem: Hearer is deciding whether to pass the various boys' teams in a raft-building competition. Action1:pass Team1, fail Team2. Action2: pass Team2, fail Team1. Action3: pass both, Action4: fail both.

b. The boys built a raft= $=\lambda \mathrm{w} . \exists \mathrm{p}[\mathrm{p} \in\{\mathrm{g}(\mathrm{w}) \in \mathrm{REL}(\mathrm{DeP})($ boys $)($ built $)(\mathrm{w}) \& \mathrm{~g}(\mathrm{w})$ built a raft $\} \& \mathrm{p}=1]$

c. Pool of propositions REL chooses from:

a

$\uparrow(\mathrm{a} \sqcup \mathrm{b} \sqcup \mathrm{c} \sqcup \mathrm{d})$ built $\approx$ Team $1=\uparrow(\mathrm{a} \sqcup \mathrm{b} \sqcup \mathrm{c} \sqcup \mathrm{d}) \sqcup \mathrm{Team} 2=\uparrow(\mathrm{a} \sqcup \mathrm{b} \sqcup \mathrm{c} \sqcup \mathrm{d})$ built $a \sqcup b \sqcup c$ built.raft $\quad a \sqcup b \sqcup d$ built.raft $\uparrow(\mathrm{a} \sqcup \mathrm{b} \sqcup \mathrm{c})$ built.raft $\approx$ Team $1=\uparrow(\mathrm{a} \sqcup \mathrm{b} \sqcup \mathrm{c}) \sqcup \mathrm{Team} 2=\uparrow(\mathrm{a} \sqcup \mathrm{b} \sqcup \mathrm{c})$ built.raft ...

$\uparrow(\mathrm{a} \sqcup \mathrm{b})$ built.raft $\approx$ Team $1=\uparrow(\mathrm{a} \sqcup \mathrm{b}) \sqcup \mathrm{Team} 2=\uparrow(\mathrm{a} \sqcup \mathrm{b})$ built.raft $\ldots$ $\mathrm{a} \sqcup \mathrm{b}$ built.raft $\mathrm{b} \sqcup \mathrm{d}$ built.raft $\mathrm{a} \sqcup \mathrm{c}$ built.raft ...

$\mathrm{a} \sqcup \mathrm{b} \sqcup \uparrow(\mathrm{c} \sqcup \mathrm{d}) \mathrm{built} . \mathrm{raft} . . . \uparrow(\mathrm{a} \sqcup \mathrm{b}) \sqcup \mathrm{c} \sqcup \mathrm{a}$ built.raft ... a built.raft $\approx$ Team $1=\mathrm{a} \sqcup$ Team $2=$ a built.raft

b built.raft $\approx$ Team $1=$ b $\sqcup$ Team $2=$ b built.raft

$\cdots$

$\uparrow(\mathrm{a} \sqcup \mathrm{b}) \sqcup \uparrow(\mathrm{c} \sqcup \mathrm{d})$ built.raft $\uparrow(\mathrm{a} \sqcup \mathrm{c}) \sqcup \uparrow(\mathrm{b} \sqcup \mathrm{d})$ built.raft

$\uparrow(\mathrm{a} \sqcup \mathrm{b} \sqcup \mathrm{c}) \sqcup \mathrm{d}$ built.raft $\uparrow(\mathrm{a} \sqcup \mathrm{b} \sqcup \mathrm{c}) \sqcup \uparrow(\mathrm{c} \sqcup \mathrm{d})$ built.raft ... 
d. Some possible interpretations:

i. Andy built a raft \& Bill did, \& Chris\&Dan (as a group) did too.

(): ii. Team 1 built a raft $\&$ team 2 built a raft.

iii. Andy built a raft \& Bill did \& Chris did \& Dan did.

Suppose that exact composition of the teams is not known (but it is known that there are two boy-made teams in the competition). Interpretation [i] fails to resolve the question of the teams' performance. Both the interpretations [ii] and [iii] resolve the problem, and so are relevant. But [iii] is over-informative, and so [ii] is the interpretation of choice.

The remaining propositions in the set represent the set of speaker's epistemic possibilities, i.e., the speaker's uncertainty about the composition of the teams. ${ }^{8}$ In this framework, speaker's uncertainty about the exact distribution of individuals into "packages" will prevent REL from eliminating the propositions that involve the correct level of distributivity. The existential quantification over propositions will then capture the speaker's inability to choose among the remaining propositions. ${ }^{9}$

The framework also opens up a possibility of having non-maximal intermediate-level interpretations, impossible to derive in Landman 1989 or Theory IV (Landman's 1996 recasting of Schwarzschild 1991). Empirically, such readings do arise sometimes. For instance, in an international soccer competition, where men's and women's teams from various countries compete, an observer can say "The African men won" to mean that some of the men's teams from Africa won (say, Ghana and Tunisia), even if some lost (say, Togo).

\section{Conclusions}

\subsection{Back to Questions}

The DT approach takes as its base a weak semantics for definite plurals, and builds in pragmatic factors to derive stronger truth-conditions for sentences with definites. Speakers' estimates for each other's beliefs, goals, and available actions are incorporated into selection of maximally relevant propositions with subparts of the plural definite $(6,7)$. The framework derives the (non)maximality and distributivity patterns and makes correct predictions about speaker knowledge.

\footnotetext{
${ }^{8}$ Thanks to Chris Potts for pointing this out.

${ }^{9}$ In a more intensionalized framework, REL would output not the individuals (boys), but functions $\mathrm{g}(\mathrm{w})$ that, for each world, give correct "cover" (the two boys' teams) in that world. The resulting set of propositions that remain after REL applies is then a singleton: $\{$ teams $(w)$ built.raft\}. It's not immediately clear if such system permits non-maximal intermediate-distributive readings.
} 
This framework makes interpretation of definite plurals parallel to that of questions (van Rooy 1999), where speakers' estimates for each other's Decision Problems are incorporated into selection of the set of maximally relevant answers.
A. Where do you live?
b. $\{\lambda v[g \in \operatorname{REL}($ place $)(\mathrm{P})(\mathrm{v})]: \mathrm{w} \in \mathrm{W} \& \mathrm{~g} \in \mathrm{REL}($ place $)(\mathrm{P})(\mathrm{w})\}$, where $\mathrm{P}=\lambda \mathrm{w} \lambda \mathrm{x}$ You live in $\mathrm{x}$ in $\mathrm{w}$
c. Possible partitions: i. \{You live in USA, You live in France,,..$\}$
ii. \{You live at 1 High St. Enid OK,...\}

d. Decision Problem1: Questioner interested in background of hearer to select a conversation topic: US sports (Action1), French cuisine (Action2), ...

Decision Problem2: Questioner has to mail hearer a package to (actions corresponding to different addresses)

a. Where can I buy an Italian newspaper?

b. $\{\lambda v[g \in \operatorname{REL}($ place $)(\mathrm{P})(\mathrm{v})]: \mathrm{w} \in \mathrm{W} \& \mathrm{~g} \in \operatorname{REL}($ place $)(\mathrm{P})(\mathrm{w})\}$, with $\mathrm{P}=\lambda \mathrm{w} . \lambda \mathrm{x}$.Italian newspaper sold at $\mathrm{x}$ in $\mathrm{w}$

c. Possible sets: i. $\{\{\mathrm{u}=$ It.news sold at the palace $\},\{\mathrm{v}=$ It.news sold at the station $\},\{\mathrm{w}=$ It.news sold at the palace $\&$ the station $\}\}$ ii. $\{\{\mathrm{u}, \mathrm{w}\},\{\mathrm{v}, \mathrm{w}\}\}$

Decision Problem1: In Amsterdam, questioner wants to read news in Italian by buying the newspaper at the station (Action1), or at the palace (Action2)

Decision Problem2: In Amsterdam, questioner is surveying availability of Italian press for a travel website (actions corresponding to writing down different sets of places where Italian press is sold)

In the same way as it does for plurals, this framework results in mention-all question interpretations when in each world, the optimally-relevant answers are singleton sets. Exactly when several answers are equally optimally-relevant, the REL will yield a mention-some interpretation for the questions.

\subsection{Overt Distributivity and Maximality Operators}

Not all sentences with definite plurals are vague. Operators like all or each force maximal (and, in the case of each, fully distributive) interpretations in sentences with definite plurals (26), compare with (27).

(26) a. All the boys surrounded the castle.

b. The boys built a raft each.

a. The boys surrounded the castle.

b. The boys built a raft. 
How do these lexical items achieve this effect? Theoretically, two mechanisms are possible. First, the lexical operator (all or each) can work in the semantics to limit or change the available interpretations. Another possibility is for these operators to contribute information about extra-linguistic factors (affecting the Decision Problem), so that REL, as a result, will give the desired interpretations.

Winter $(1998,2002)$ adopts the former approach to these operators, while Brisson (1998) pursues the latter. Empirical data suggests that no extra-linguistic factors can force a maximal interpretation in collective sentences like (27a). So, it cannot be that all affects the context to force a maximal interpretation in (26a).

This is matched by inability of REL, on our account, to distinguish between maximal and non-maximal interpretations for collective predication. Thus, I adopt Winter's approach, in which all (and each) work in the semantics to introduce universal quantification (and full distributivity) over atoms in the NP.

In Winter's (2002) framework NP and VP denotations can denote sets of atomic entities, or sets of sets of entities. He defines all as a composite determiner $d$ fit(every), where every has the usual denotation (a relation between sets of atomic entities), while $d$ fit operation is defined as in (28).

(28) Let $D$ be a relation between sets of elements in a domain $E$ of atoms. The operator $d f i t$ (determiner fitting) maps $D$ to a relation $d$ fit $(D)$ between sets of sets of atoms in $p(E)$, which is defined as follows:

For any two sets $A, B \in p(E)$, the relation $(\operatorname{dfit}(D))(A, B)$ holds iff the relation $D(\cup A, \cup(A \cap B)$ holds.

Translating this to the notation used here (with groups and sums), and making this specific to all, we get (29a). Without REL, this would produce the denotation in (29b) for (26a).

a. $\quad$ all $=d f i t($ every $)=\lambda \mathrm{P} . \lambda \mathrm{Q}$. Every $(\sqcup \downarrow \mathrm{P}, \sqcup(\downarrow \mathrm{P} \sqcap \downarrow \mathrm{Q}))$

b. all the boys surrounded the castle $=$ $=$ Every $\left(\sigma x .{ }^{*} \operatorname{boy}(\mathrm{x}), \sqcup\left(\sigma \mathrm{x} .{ }^{*} \operatorname{boy}(\mathrm{x}) \sqcap \sigma \mathrm{x}\right.\right.$. surrounded.castle $\left.\left.(\mathrm{x})\right)\right)$ $=$ Every boy participated in a set of boys that surrounded the castle

As in the case without all, REL will produce a set of propositions in the end (because of the type mismatch, combining via function combination, as before). This will be a singleton set. Translating Winter's framework to the notation used in this paper, with some adjustments we will get (30)

$$
\begin{aligned}
& \lambda \text { Q.Every }(\sqcup \downarrow N P, \sqcup(\downarrow N P \sqcap \downarrow Q))(\lambda x . x \in R E L(D e P)(V P)(N P) \& V P(x))= \\
& =\text { Every }(\sqcup \downarrow N P, \sqcup(\downarrow N P \sqcap(\{\downarrow x: x \in \operatorname{REL}(\operatorname{DeP})(\mathrm{VP})(\mathrm{NP}) \& V P(x)\}))
\end{aligned}
$$

This results in the denotation for (26a) given in (31a) and paraphrased in (31b): 
(31)

a. $\operatorname{Every}\left(\sigma x .{ }^{*} \operatorname{boy}(\mathrm{x}), \sqcup\left(\sigma x .{ }^{*} \operatorname{boy}(\mathrm{x}) \sqcap \sqcup\{\downarrow d \mathrm{~d}: \mathrm{d} \in \mathrm{AT}(\downarrow \mathrm{x})\right.\right.$ : $\left.\left.\left.\mathrm{x} \in \operatorname{REL}(\operatorname{DeP})(\operatorname{sr} . \operatorname{csl})\left(\sigma \mathrm{x} .{ }^{*} \operatorname{boy}(\mathrm{x}) \& \operatorname{sr} . \operatorname{csl}(\mathrm{x})\right)\right\}\right)\right)$

b. Every boy participated in a set of relevant boys that surrounded the castle.

Notice that (31b) is equivalent to (29b), except that (31b) can be taken to contribute an additional statement about the Decision Problem (namely, that the proposition with "all the boys" is optimally relevant).

\subsection{Some Further Issues}

The Decision Theory approach, unifying the interpretation of interrogatives and definite plurals, lays the groundwork for explaining the parallel behavior of questions and plurals with respect to QVE (Lahiri 1991, 1998, Williams 2000, Beck and Sharvit 2002) (32).

(32) a. For the most part, Al knows about which kids are drunk

b. For the most part, Al hates the kids on his block

c. For the most part, Al knows where the kids are hiding

Quantification over parts can apply to wh-phrase denotations (32a) as well as to definite plurals (32b). Sometimes, it can apply to either, depending on the context (Williams 2000) (32c). A framework using similar mechanisms for interpreting definite plurals and wh-phrases is a prerequisite for addressing this data.

\section{References}

Bar-Hillel, Yehoshua and Rudolf Carnap: 1953, in W. Jackson 'Semantic Information', in Communication Theory, Papers read at a Symposium on "Applications of Communication Theory", Held at the Institution of Electrical Engineers, London, September 22nd-26th, 1952, Butterworth Scientific Publications, London, 503-511.

Beck, Sigrid and Yael Sharvit: 2002, 'Pluralities of Questions', Journal of Semantics 19(2), 105-115.

Brisson, Christine: 1998, Distributivity, Maximality, and Floating Quantifiers. Doctoral Dissertation, Rutgers University.

Dowty, David: 1987, 'Collective Predicates, Distributive Predicates, and All', in F. Marshall, A. Miller and Z. Zang (eds.) Proceedings of ESCOL '86, 97115.

Grice, H. Paul: 1975, 'Logic and Conversation', in P. Cole and J. Morgan (eds.) Syntax and semantics 3: Speech Acts, Academic Press, New York, 31-58. 
Keshet, Ezra. 2006. 'Scalar Implicatures with Alternative Semantics', this volume.

Kratzer, Angelika: 2003. 'Cumulativity as a Possible Universal', Ch. 4 of The Event Argument and the Semantics of Verbs. Ms, University of Massachusetts, Amherst.

Kratzer, Angelika and Junko Shimoyama: 2002, 'Indeterminate Phrases: the View from Japanese', in Y. Otsu (ed.) Proceedings of the Third Tokyo Conference on Psycholinguistics, Hituzi Syobo, Tokyo.

Krifka, Manfred: 1996, 'Pragmatic Strengthening in Plural Predications and Donkey Sentences', in T. Galloway and J. Spence (eds.) Proceedings of SALT VI, CLC Publications, Cornell University, Ithaca.

Lahiri, Utpal: 1991, Embedded Interrogatives and the Predicates that Embed Them. Doctoral Dissertation, MIT.

Lahiri, Utpal: 1998, Questions and Answers in Embedded Contexts. Ms., UC Irvine.

Landman, Fred: 1989. 'Groups, I', Linguistics and Philosophy 12, 559-605.

Landman, Fred: 1996, 'Plurality', in S. Lappin (ed.) The Handbook of Contemporary Semantic Theory, Blackwell, Cambridge, 425-457.

Lasersohn, Peter: 1990, A Semantics for Groups and Events, Garland Publishing, New York.

Link, Godehard: 1983, 'The Logical Analysis of Plurals and Mass Terms: A Lattice-Theoretic Approach', in R. Bauerle, C. Schwarze, and A. von Stechow (eds.) Meaning, Use, and Interpretation of Language, de Gruyter, Berlin, 302-323.

Lønning, Jan T.: 1987, 'Collective Readings of Definite and Indefinite Noun Phrases', in P. Gärdenfors (ed.) Generalised Quantifiers, Reidel, Dordrecht, 203-235.

Merin, Arthur: 1999, 'Information, Relevance and Social Decisionmaking: Some Principles and Results of Decision-Theoretic Semantics', in L. Moss, J. Ginzburg, and M. de Rijke (eds.) Logic, Language, and Computation Vol. 2, CSLI, Stanford, 179-221.

Raiffa, Howard: 1968, Decision Analysis: Introductory Lectures on Choices under Uncertainty, Addison-Wesley, Reading, MA.

van Rooy, Robert: 1999, 'Questioning to Resolve Decision Problems', in P. Dekker (ed.), Proceedings of the Twelfth Amsterdam Colloquium, ILLC, Amsterdam, 211-216.

van Rooy, Robert: 2003, 'Negative Polarity Items in Questions: Strength as Relevance', Journal of Semantics 20, 239-273.

Schwarzschild, Roger: 1991, On the Meaning of Definite Plural Noun Phrases. Doctoral Dissertation, University of Massachusetts, Amherst. 